\title{
Four-stage evolution of diabetes or whole body insulin resistance (WBIR): Debunking of the lipid-induced insulin resistance (LIIR) and proposing of the glycation-induced insulin resistance (GIIR)
}

\author{
Song Jae Lee ${ }^{1}$ and Sang Won Shin ${ }^{2 *}$ \\ ${ }^{1}$ Chungnam National University, Daejeon, South Korea; sjlee@cnu.ac.kr \\ ${ }^{2}$ Korea University, Seoul, South Korea; shinsw@kumc.or.kr \\ *Correspondence to: SJ Lee, sjlee@cnu.ac.kr
}

\begin{abstract}
Even though it has long been known that diabetes develops in distinctive stages over a long span of time, no comprehensive diabetes development model has been developed yet. Insulin resistance (IR) plays a central role in development of diabetes. A widespread belief regarding IR is that it is a global parameter affecting the whole body simultaneously by impairing merely glucose uptake in tissues. However, the analysis by a new methodology that we have named integrated approach suggests that IR not merely impairs glucose uptake in tissues but also produces tissue-specific metabolic disruptions varying widely from tissue to tissue, and that IR would not necessarily develop simultaneously over the whole body but instead develop first preferentially in the muscle tissue with a relatively low cell turnover and then progress in sequence to the subcutaneous adipose tissue to the visceral adipose tissue to the liver with higher cell turnovers. This is the most important rationale for subdividing IR into four distinct tissue-specific IRs: muscle insulin resistance (MIR), subcutaneous adipose insulin resistance (s-AIR), visceral adipose insulin resistance ( $v$-AIR), and hepatic insulin resistance (HIR). Sequential development of tissue-specific IRs, in the order of MIR, s-AIR, v-AIR, and HIR, producing tissue-specific metabolic disruptions, is nothing but the whole body insulin resistance (WBIR) evolving in four distinctively insulin-resistant stages. Four-stage evolution from rapid weight gain to visceral obesity to rapid weight loss to full-blown diabetic state not only complies well with the natural development history of diabetes, but also resolves most of controversies on diabetes or obesity. Development of the four-stage WBIR evolution model, which also refutes the entrenched notion of the lipid-induced insulin resistance (LIIR) but instead supports the glycation-induced insulin resistance (GIIR) proposed in this study, may possibly be considered a breakthrough in study of diabetes as well as obesity.
\end{abstract}

Keywords: obesity, obesity paradox, diabetes, insulin resistance (IR), whole body insulin resistance (WBIR), tissue-specific insulin resistance; muscle insulin resistance (MIR), subcutaneous insulin resistance (s-AIR), visceral adipose insulin resistance ( $v$-AIR), hepatic insulin resistance (HIR), lipid-induced insulin resistance (LIIIR), glycation-induced insulin resistance (GIIIR) 


\section{Introduction}

The aim of this study is to develop a comprehensive diabetes development model that complies well with the natural development of diabetes (1). Insulin resistance (IR) plays a central role in development of diabetes, which appears to be at the center of association among many disorders, including cardio/cerebral vasculopathy, nephropathy, neuropathy, and retinopathy. IR may be defined as the physiological state or condition, in which cells or tissues have been deranged enough as to be unable to respond properly to insulin, the major hormone that regulates the metabolism. Yet specific role of insulin is widely different from tissue to tissue (2). Consequently, metabolic disruptions due to IR would be very tissue-specific and wide-ranging (3-5), which is contrary to the common belief that IR impairs merely glucose uptake in tissues.

No less importantly, IR would not necessarily develop simultaneously over the whole body. Instead, IR more likely develops first preferentially in old cells over newly generated cells, simply because old ones would have been exposed longer to potential IR-inducing agents than new ones. The lower the cell turnover in a tissue, the larger the population of old cells in that tissue. This will translate into that tissues with a low cell turnover would generally be more susceptible to IR than tissues with a high cell turnover.

Adipose tissues are quite unique in that they frequently differentiate preadipocytes into adult adipocytes with energy storage demand increasing - adipogenesis (6). On the other hand, myogenesis in muscle tissues appears to be generally much smaller than adipogenesis (7). This implies that the average cells in muscle tissues would be much older than the average cells in adipose tissues, which translates into that muscle tissues in general would develop IR more readily than adipose tissues. Meanwhile, adipogenesis is known to be much more active in the visceral adipose tissue than in the subcutaneous adipose tissue (8), which translates into that the subcutaneous adipose tissue would develop IR more readily than the visceral adipose tissue. In the case of the liver that works also as a major detoxification center in the body, cells may be damaged and replaced rather frequently, leading to a very high cell turnover. Based on this reasoning, we postulate that in IR-inducing physiological condition, IR develop first in the muscle tissue and progress in sequence to the subcutaneous adipose tissue, to the visceral adipose tissue, and to the liver. It would be noteworthy that the postulation disputes the common notion that IR is a global parameter affecting the whole body simultaneously.

If IR indeed develops in sequence from the muscle tissue to subcutaneous adipose tissue to visceral adipose tissue to liver, producing tissue-specific metabolic disruptions, it should be subdivided better into four distinct tissue-specific IRs: muscle insulin resistance (MIR), subcutaneous adipose insulin resistance (s-AIR), visceral adipose insulin resistance ( $v$-AIR), and hepatic insulin resistance (HIR). Sequential development of tissue-specific IRs in the order of MIR, s-AIR, v-AIR and $H I R$, as depicted in Fig 1, would amount to nothing but the whole-body insulin resistance (WBIR) evolving in four distinctively insulin-resistant (IR) stages, denoted by IR-I, IR-II, IR-III, IR-IV, 
respectively (9).

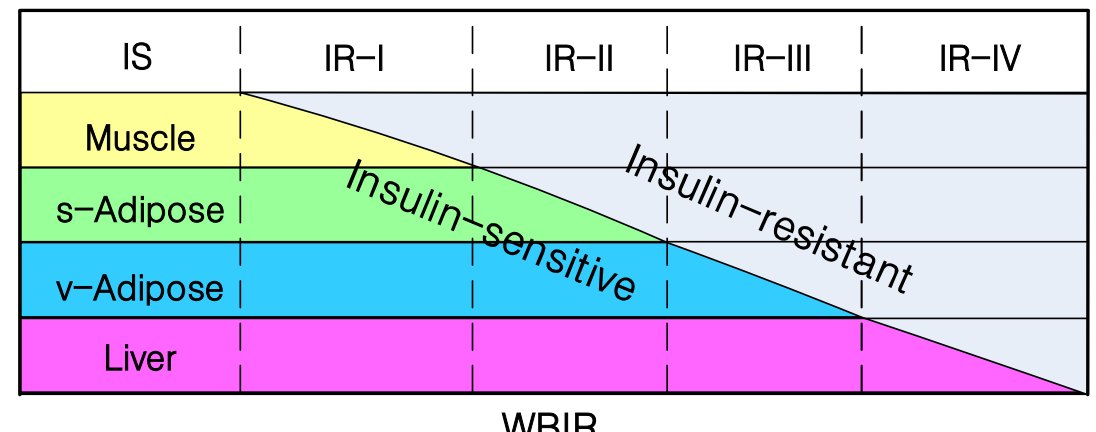

Fig 1. WBIR evolves in four distinctive stages as the tissue-specific IRs develop in sequence in the order of MIR, s-AIR, v-AIR, and HIR.

WBIR evolution would inevitably culminate in the development of diabetes in the IR-IV stage, in which all of the four major tissues exhibit simultaneously severe IR and therefore PG would elevate severely enough to be indicative of diabetes. This means in other words that the four-stage WBIR evolution model amounts, in fact, to the four-stage diabetes development model.

Actually, Saad et al. proposed a two-step model for diabetes development (10). However, the model lacking the concept of tissue-specific IRs developing in sequence among various tissues could not explain, for instance, that individuals experience, over the course of diabetes development, metabolic evolution from rapid weight gain to visceral obesity to rapid weight loss to full-flown diabetic state. By contrast, the metabolic evolution predicted by the four-stage WBIR evolution model complies well with natural development history of diabetes (1). In addition, the model not only resolves most of the controversies on diabetes or obesity, including visceral obesity, obesity paradox, and dawn phenomenon that have long remained a puzzle, but also provides valuable insights on the true nature of the association between obesity and diabetes.

Yet the four-stage WBIR evolution model itself does not tell anything about IR-inducing agents or mechanisms, simply because it does not presume any particular IR-inducing agent or mechanism. A widespread belief regarding IR is that lipids induce IR. A core argument of the so-called the lipid-induced insulin resistance (LIIR) is that fat mass or obesity induces IR either directly or indirectly by contributing excess fatty acids (FAs) to the plasma so that resultant elevated plasma fatty acids (PFAs) somehow interfere the insulin action in cells or tissues (11).

However, basics of physiology and metabolism suggests that weight gaining or obesity is a kind of proper metabolic response of the body or the adipose tissues in particular exposed to hyperglycemia and in fact has an effect of alleviating hyperglycemia. Then, how can weight gaining or obesity induce IR and at the same time alleviate hyperglycemia that is a prominent symptom of IR? A revealing observation here is that newly diagnosed diabetics lose weight rapidly, only to gain some weight back only if treated by anti-diabetic medications, such as insulin or oral hypoglycemic agents (OHAs) (12). This suggests that adipose tissues in diabetics may be unable to respond 
properly to hyperglycemia by accumulating fat mass. This in turn suggests conversely that only healthy adipose tissues are able to support weight gaining or obesity. And this may also explain why lean individuals often exhibit higher mortality compared overweight or obese individuals obesity paradox (13). If weight gaining or obesity indeed has an effect of alleviating hyperglycemia, it would hardly be a risk factor per se though it would still hardly be beneficial overall for health. This suggests strongly that behind weight gaining or obesity lies an independent risk factor, which is nothing but the hyperglycemia itself. This in turn means that it is not proper to find fault with obesity, rather than with hyperglycemia.

The reasoning above largely refutes the entrenched notion of the lipid-induced insulin resistance (LIIR) or indicates that the LIIR is not compatible, at least, to the four-stage WBIR evolution model. It would then be essential to introduce a proper IR inducing mechanism that complies well with the four-stage WBIR evolution model. An important aspect of the four-stage WBIR evolution is that plasma glucose $(P G)$ or, more accurately, time-averaged PG elevates steadily with WBIR evolving. A reaction activated in hyperglycemic physiological condition is glycation or non-enzymatic glycosylation, in which glucose or its glycolytic derivatives, such as glyoxal and methylglyoxal, react non-enzymatically with proteins and form covalently bonded adducts $(14,15)$. Glycation adducts may neutralize some of the charges on the amino acid residues (16), thereby significantly changing the electrostatic forces between residues and therefore significantly altering the protein conformation as well. The protein with both the charge distribution and conformation disrupted significantly as a result of glycation can hardly function properly.

For example, insulin receptors or other proteins involved in downstream insulin signaling may also be glycated severely enough as to render them unable to respond properly to insulin. The cells or tissues unable to respond properly to insulin as a result of glycation would exhibit nothing but glycation-induced insulin resistance (GIIR). Most importantly, GIIR would enhance hyperglycemia further by impairing glucose uptake into tissues, which would in turn enhance glycation reaction and GIIR further, thereby establishing a positive feedback loop in which hyperglycemia and GIIR enhance each other in a vicious circle. In reality, the four-stage evolution model predicts that PG elevates at an ever faster pace with WBIR evolving. This suggests strongly that GIIR is the very IR-inducing mechanism behind WBIR evolution.

The most important implication of GIIR underlying the WBIR evolution is that hyperglycemia is not only the effect of WBIR evolution but also the main driving force for WBIR evolution. Then, an obvious approach to prevent, delay, or reverse WBIR evolution or diabetes development will be to alleviate hyperglycemia, which is in fact a primary goal of most diabetes management or treatment plans. And an obvious approach to achieve alleviation of hyperglycemia will be to reduce postprandial glycemic load by curtailing carbohydrate intake. 


\section{Materials and Methods}

WBIR evolution proceeding in general very slowly over years or even decades may not be analyzed easily by simple experimental studies, especially conducted over a relatively short span of time. In addition, the pace of WBIR evolution in individuals would be widely different, depending on the concentration of potential IR-inducing agents. Thus, the subjects recruited for an experiment would be more likely to belong to different stages of WBIR evolution, which renders metabolic data obtained much more complex and makes it much more difficult to analyze them.

WBIR evolution may be analyzed better by integrated approach that we have newly introduced for this study. We believe that integrated approach had already been used extensively especially by the nineteenth century evolutionists, including Charles Darwin, to develop the evolution theory. They had carefully observed an enormous amount of data achieved by experiments done by the Nature over immensely long period of time and then derived plausible theories or principles, without actually performing themselves experiments that would have generally required raising animals or growing plants for numerous generations.

The single most important task in integrated approach is to build the integrated pool of resources, as depicted in Fig 2, which consists of two parts: (1) the resource of basics that includes basics of physiology/metabolism, basic theories/principles, and simple logics/reasonings, and (2) the resource of a wide range of consistent experimental data that support or comply well with the resource of basics - the experimental data complying well with the resource of basics may be called the consistent experimental data. Initially, the resource of basics would be obtained mainly by reviewing textbooks on physiology or metabolism and then expanded ever more by surveying literatures across the wide range of disciplines. And the resource of consistent experimental data would be obtained mainly by surveying experimental studies and incorporating only the experimental data that support or comply well with the resource of the basics. Most importantly, the integrated pool of resources will be expanded and refined ever more by continually surveying literatures across the wide range of disciplines. 


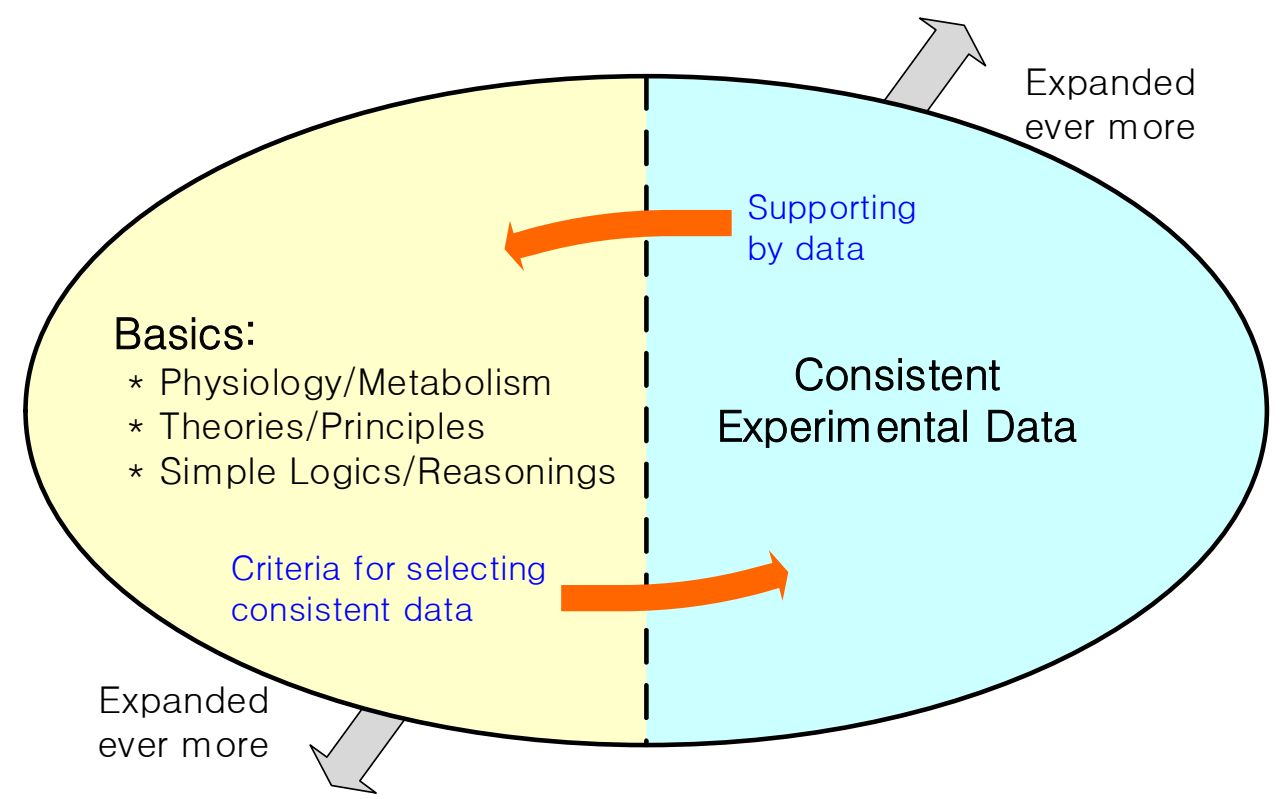

Fig 2. Integrated approach is based on integrated pool of resources, consisting of the resource of basics and the resource of consistent experimental data complying well with the resource of basics.

A prevailing trend in current medical researches may be that they are mostly done by experiments that largely ignore the resource of basics and mainly measure associations between metabolic variables. However, the associations alone, with the basics largely ignored, would not tell much about the causality and often lead to the fallacy of assuming the association as the causality, which may be one of the most important reasons for the cause-effect confusions frequently observed in medical researches. On the other hand, the conclusions or theories attained by simple experimental studies may not be very reliable, simply because they are drawn based on a rather limited amount of data obtained by only a few number of experiments that are usually focused narrowly only on a few metabolic variables.

By contrast, in the case of researches based on integrated approach, fundamental theories or principles are derived first relying mainly on the resource of the basics and then validated by a wide range of consistent experimental data achieved across the wide range of disciplines. Consequently, the theories or conclusions derived based on integrated approach would generally be much more reliable than those attained by simple experimental studies. The more reliable conclusions or principles achieved by the integrated approach would help rectify the cause-effect confusions or misconceptions frequently observed in medical researches. This may conversely explain why the four-stage WBIR evolution or diabetes development model developed based on integrated approach not only resolves most of the controversies on obesity or diabetes, including visceral obesity, obesity paradox, and dawn phenomenon that have long remained a puzzle, but also provides valuable insights on the true nature of the association between obesity and diabetes.

Lastly, we believe that modern researchers are in a much better position than the nineteenth 
century scientists to exploit the integrated approach, simply because we have volumes of textbooks on physiology, metabolism, biology, or biochemistry as well as virtually unlimited amount of experimental data readily available to validate our reasoning or theorization.

\section{Results}

\section{Tissue-Specific IRs}

Metabolic disruptions due to tissue-specific IRs may easily be deduced by identifying the tissue-specific roles of insulin and largely verified by referring to the metabolic disruptions observed in some genetically-engineered tissue-specific IR models, like MIRKO (muscle-tissue specific insulin receptor knock-out) (3), FIRKO (fat-tissue specific insulin receptor knock-out) (5), and LIRKO (liver-specific insulin receptor knock-out) mice (4). Obviously, the MIRKO, FIRKO, and LIRKO mice would be unable to initiate insulin signaling in the muscle tissue, adipose tissue, and liver, respectively, and therefore inevitably exhibit MIR, AIR, and HIR, respectively. Key aspects of the tissue-specific IRs are summarized in Table 1. A noteworthy point here is that metabolic disruptions due to tissue-specific IRs are wide-ranging and rather different from tissue to tissue, which disputes the common notion that IR merely impairs glucose uptake in tissues.

Table 1. Key aspects of tissue-specific IRs.

\begin{tabular}{|c|c|}
\hline MIR & $\begin{array}{l}\text { - Disruption of insulin signaling in the muscle tissue } \\
\text { - Impairment of insulin-mediated muscle glucose uptake and muscle glycogenesis } \\
\text { - Elevating PPG significantly } \\
\text { - Muscle glucose uptake mediated by muscle contraction or exercise is largely spared from MIR }\end{array}$ \\
\hline S-AIR & $\begin{array}{l}\text { - Disruption of insulin signaling in the subcutaneous adipose tissue } \\
\text { - Impairment of the subcutaneous adipose glucose uptake: } \\
\text { - Elevating PPG significantly } \\
\text { - Elevating PI (PPI and FPI) significantly (as long as insulin secretory response is not impaired) } \\
\text { - Subcutaneous adipose tissue undergoes uninhibited lipolysis and fails to entrap the FAs released from TRLs } \\
\text { - The excess PFAs shunned by the subcutaneous adipose tissue likely to be entrapped by the visceral } \\
\text { adipose tissue } \\
\text { - Elevating PFA only modestly } \\
\text { - Subcutaneous adipose tissue losing fat mass rapidly }\end{array}$ \\
\hline V-AIR & $\begin{array}{l}\text { - Disruption of insulin signaling in the visceral adipose tissue } \\
\text { - Impairment of visceral adipose glucose uptake: } \\
\text { - Elevating PPG significantly } \\
\text { - Elevating PI (PPI and FPI) significantly (as long as insulin secretory response is not impaired) } \\
\text { - Impairment of v-ADNL and visceral fat accumulation in the fed state } \\
\text { - Visceral adipose tissue undergoes uninhibited lipolysis and fails to entrap the FAs released from TRLs } \\
\text { - Elevating PFA severely } \\
\text { - Enhancing ectopic fat deposition } \\
\text { - Elevating PG severely as well (glucose-FA cycle: PFA elevation restricting PG utilization) } \\
\text { - Visceral adipose tissue losing fat mass rapidly }\end{array}$ \\
\hline
\end{tabular}




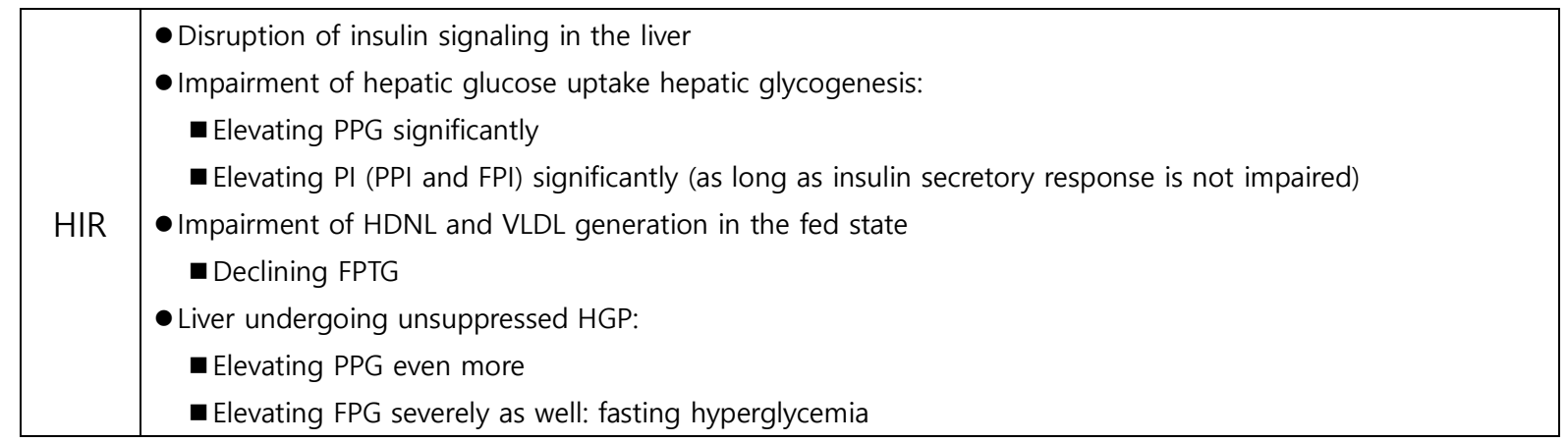

MIR: MIR or disruption of muscle insulin signaling as observed in the MIRKO mice (3) impairs glucose uptake into muscle tissues, not only restricting significantly muscle glucose oxidation as well as muscle glycogenesis but also elevating postprandial plasma glucose (PPG) significantly. The elevation of PPG will in turn enhance postprandial insulin secretion, significantly elevating postprandial plasma insulin (PPI).

It is rather surprising, however, that the hyperglycemia observed in the MIRKO mice exhibiting MIR appears to be relatively mild (3), especially considering that muscle tissues are generally responsible for more than $70 \%$ of glucose disposal in the body. This may serve as evidence that muscle tissues have another glucose uptake pathway that is mediated - independently of insulin by muscle contraction or exercise (17) and therefore largely spared from MIR $(18,19)$.

AIR: AIR or disruption of adipose insulin signaling as observed in the FIRKO mice (5) not only impairs adipose glucose uptake, elevating PPG and PPI significantly, but also disrupts severely the adipose metabolism. It may be necessary to discuss briefly the healthy adipose metabolism, not disrupted by AIR. In the case of the fed state regulated by relatively high postprandial plasma insulin (PPI), adipose tissues actively take up PG and mostly convert the glucose, via adipose de novo lipogenesis (ADNL) pathway, into fatty acids (FAs) $(20,21)$. In addition, they also entrap the FAs released from triglyceride-rich lipoproteins (TRLs), such as very low-density lipoproteins (VLDLs) and chylomicrons (22). The FAs either synthesized inside or fluxed from the plasma will be esterified mostly into fats for storage. On the other hand, in the case of the fasted state regulated by relatively low fasting plasma insulin (FPI), adipose lipases, such as hormone sensitive lipase (HSL) and adipose triglyceride lipase (ATGL), will not be inhibited strongly enough (23). Consequently, adipose tissues undergo lipolysis and release the resultant FAs into the plasma, thereby elevating PFAs significantly. The significantly elevated PFAs subsequently diffuse into other tissues or organs $(24,25)$ and mostly are utilized as primary fasting energy substrates. The healthy adipose tissues that are involved in the FA-to-fat anabolism in the fed state and the fat-to-FA catabolism in the fasted state may be regarded not merely as a fat storage depot but, more accurately, as a fatty acid (FA) reservoir.

If adipose tissues develop AIR, however, they can hardly work as a FA reservoir simply because insulin is no longer able to inhibit adipose lipases, which would make adipose tissues not only undergo uninhibited lipolysis, regardless of PI level, but also fail to entrap the FAs released from 
TRLs, thereby elevating plasma fatty acid (PFA) severely. In this sense, severe elevation of PFA may be considered a hallmark of HIR. Furthermore, the AIR-associated uninhibited lipolysis would also deplete rapidly the fat mass stored in the adipose tissue, thereby leading to rapid weight loss.

The excess PFAs shunned or released by the AIR-affected adipose tissues would have no choice but to diffuse into other tissues or grans, notably muscle tissues and liver, and subsequently esterify into ectopic fats (26). This would explain why the A-ZIP/F-1 transgenic mice, lacking white adipose tissue to work as a FA reservoir, also exhibit a significant amount of ectopic fats (27), exactly like the FIRKO mice. In addition, severe elevation of PFA would inevitably restrict utilization of PG as competing energy substrate - Randle cycle or glucose-FA cycle $(28,29)$ - thereby effectively elevating PG significantly. An important point here is that PG seems to be inherently at a significant disadvantage in competing with PFAs. Glucose that is polar or hydrophilic requires special plasma membrane transporters, like GLUT4 and GLUT2, as well as insulin mediation, for transporting across the plasma membrane of hydrophobic lipid bilayer (30). By contrast, FAs that are nonpolar or hydrophobic diffuse rather freely into cells $(24,25)$. This will be why PFAs are preferentially utilized over PG in severe diabetics with both PG and PFA simultaneously elevated severely (31).

The reasoning that AIR inevitably elevates PG as well as PFA suggests conversely that healthy AIR-free adipose tissues are essential for normalizing PG or glucose homeostasis as well (32). And that AIR inevitably depletes rapidly the adipose fat mass suggests conversely that only healthy AIR-free adipose tissues are able to support weight gaining or obesity. An important point here is that weight gaining is in itself an indication that adipose tissues are working effectively as a FA reservoir to contain FAs in the form of fats, effectively lowering PG as well as PFA. It is quite revealing that treatment of hyperglycemia by anti-diabetic medications (ADMs), such as insulin or oral hypoglycemic agents (OHAs), often leads to weight gain (12). On the other hand, rapid weight loss associated with AIR would have an effect of elevating PG as well as PFA.

A noteworthy point in Table 1 is that AIR has been divided into the subcutaneous adipose insulin resistance (s-AIR) and visceral adipose insulin resistance ( $v$-AIR), simply because we have assumed that the subcutaneous adipose tissue develops AIR earlier than the visceral adipose tissue. Most importantly, the metabolic disruptions due to s-AIR would be rather modest as long as the visceral adipose tissue is largely free from v-AIR and thus able to bear the burden left by the subcutaneous adipose tissue.

HIR: HIR or disruption of hepatic insulin signaling as observed in the LIRKO mice (4) impairs hepatic glucose uptake, not only elevating PG significantly but also restricting significantly both the hepatic de novo lipogenesis (HDNL) and hepatic glycogenesis. In addition, disruption of hepatic insulin signaling would also prevent insulin from suppressing hepatic glucose production (HGP). The unsuppressed HGP associated with HIR, regardless of PI level, would elevate PG severely not only in the fed state but also in the fasted state (33). Thus, fasting hyperglycemia can be considered a hallmark of HIR. 
A supporting evidence that HIR indeed leads to fasting hyperglycemia may be found in the so-called dawn phenomenon regularly observed in diabetics treated by ADMs (34). PI as well as ADMs would generally wane gradually overnight, thereby allowing HGP to reach a maximum before the next administration of ADMs usually in the morning. On the other hand, exercise-mediated glucose uptake into skeletal muscles would obviously be minimal during the sleep or rest. The combination of the maximal HGP and minimal exercise-mediated skeletal muscle glucose uptake in the early morning hours would lead to severe elevation of PG - dawn phenomenon (35).

Another important point regarding HIR is that the associated impairment of hepatic de novo lipogenesis (HDNL) would inevitably restrict VLDL generation and its secretion to the plasma, eventually declining significantly fasting plasma triglyceride (FPTG) (4). The decline of FPTG associated with HIR suggests conversely that HDNL is the most important determinant of the FPTG.

\section{Four-stage WBIR evolution and its validation}

As schematically depicted in Fig 1, WBIB would evolve in four distinctively insulin-resistant stages denoted by IR-I, IR-II, IR-III, and IR-IV, respectively, as tissue-specific IRs develop in the order of MIR, s-AIR, V-AIR, and HIR. The net metabolism to be observed in each stage can be estimated by cumulatively adding up - by referring to the Table 1 - all the metabolic disruptions resulting from all the tissue-specific IRs involved in that particular stage. Table 2 summarizes the important features of the metabolism estimated in each stage.

Table 2. Important features of the metabolism estimated in each of WBIR evolution stages

\begin{tabular}{|c|l|l|}
\hline \multirow{1}{*}{ IS } & $\begin{array}{l}\text { - Glucose uptake impaired in none of the major tissues } \\
\text { - Postprandial glycemic load elevates PPG only modestly } \\
\text { - Relatively low PPG stimulates insulin secretion modestly: relatively low PI (PPI, FPI) } \\
\text { - Fed state: relatively low PPI restricts more or less s-ADNL, V-ADNL, and HDNL/VLDL generation } \\
\text { - Fasted state: relatively low FPI enhances more or less s-lipolysis, v-lipolysis, and HGP } \\
\text { - Relatively high fasting energy substrates, FPFA and PG: } \\
\text { - Encouraging physical activity; hunger relaxed in the fasted state (rarely leading to overeating) } \\
\text { - Rarely overweight or obese } \\
\text { - MIR impairs glucose uptake into the muscle tissue, diverting excess PG to adipose tissues and liver } \\
\text { - PPG, PPI, FPI elevated more than in the IS stage } \\
\text { - Fed state: s-ADNL, v-ADNL, and HDNL/VLDL generation enhanced more strongly than in the IS stage } \\
\text { - Fasted state: s-lipolysis, v-lipolysis, and HGP inhibited more strongly than in the IS stage } \\
\text { - Relatively low fasting energy substrates, FPFA and PG: } \\
\text { - Discouraging physical activity; hunger enhanced in the fasted state (likely leading to overeating) } \\
\text { - Rapid weight gain: both the subcutaneous and visceral adipose tissue increasing fat mass rapidly }\end{array}$ \\
\hline
\end{tabular}




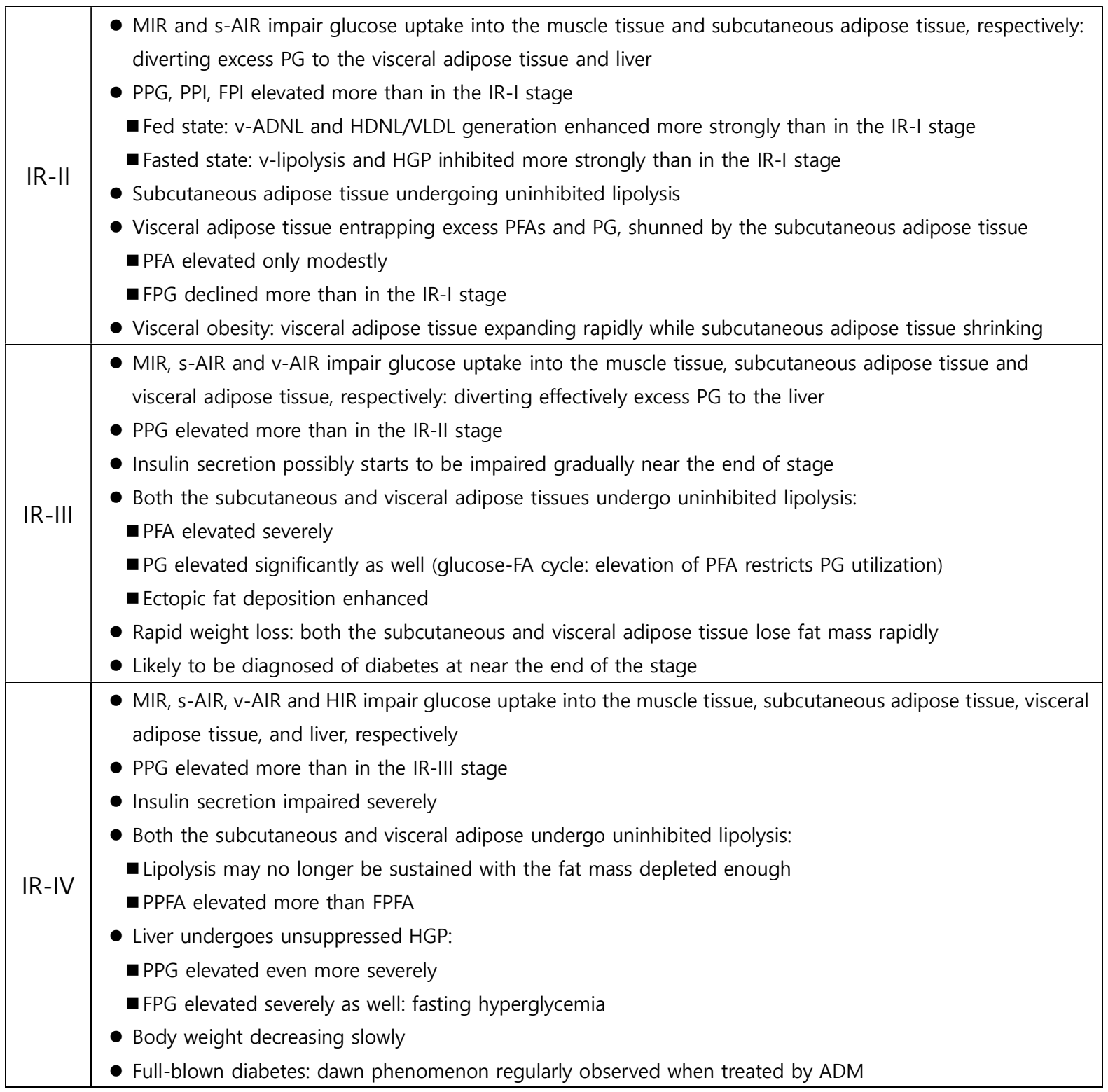

WBIR evolution would inevitably let metabolic variables evolve, over the course of diabetes development, in their own unique WBIR-dependent profiles. Table 3 describes briefly how some important metabolic variables evolve with WBIR advancing.

Tab. 3. Brief description of WBIR-dependent evolution of some important metabolic variables.

\begin{tabular}{|c|c|c|c|c|c|}
\hline Stages & IS & IR-I & IR-II & IR-III & IR-IV \\
\hline IRs involved & None & MIR & MIR, s-AIR & MIR, s-AIR, v-AIR & MIR, s-AIR, v-AIR, HIR \\
\hline PPG & $P P G_{o}$ & \multicolumn{2}{|c|}{ Elevates steadily } & Elevates steeply & Elevates more steeply \\
\hline Insulin Sec. & Moderate & \multicolumn{3}{|c|}{ Increases steadily } & Saturates then decreases \\
\hline PPI & $P P I_{o}$ & \multicolumn{3}{|c|}{ Elevates steadily } \\
\hline
\end{tabular}




\begin{tabular}{|c|c|c|c|c|c|}
\hline FPI & $F P I_{o}$ & \multicolumn{3}{|c|}{ Elevates steadily } & Saturates then declines \\
\hline $\mathrm{s}-\mathrm{ADNL}$ & Moderate & Increases & \multicolumn{3}{|c|}{ Decreases gradually } \\
\hline v-ADNL & Moderate & \multicolumn{2}{|c|}{ Increases steadily } & \multicolumn{2}{|c|}{ Decreases gradually } \\
\hline PPFA & $P P F A_{o}$ & Declines & Elevates modestly & \multicolumn{2}{|c|}{ Elevates steeply } \\
\hline HDNL & Moderate & \multicolumn{3}{|c|}{ Increases steadily } & Decrease gradually \\
\hline FPTG & $F P T G_{o}$ & \multicolumn{2}{|c|}{ Elevates steadily } & Elevates steeply & Declines gradually \\
\hline HGP & Moderate & \multicolumn{3}{|c|}{ Decreases gradually } & Increases steeply \\
\hline FPG & $F P G_{o}$ & \multicolumn{2}{|c|}{ Declines gradually } & Elevates gradually & Elevates steeply \\
\hline $\mathrm{HbA1c}$ & $H b A 1 c_{o}$ & \multicolumn{2}{|c|}{ Elevates steadily } & Elevates steeply & Elevates more steeply \\
\hline s-Lipolysis & Moderate & Decreases & \multicolumn{3}{|c|}{ Increases gradually, saturates, then decreases } \\
\hline v-Lipolysis & Moderate & \multicolumn{2}{|c|}{ Decreases steadily } & \multicolumn{2}{|c|}{ Increases gradually, saturates, then decreases } \\
\hline FPFA & $F P F A_{o}$ & Declines & Elevates modestly & Elevates steeply & Saturates then declines \\
\hline Body Weight & Healthy lean & $\begin{array}{l}\text { Increases } \\
\text { rapidly }\end{array}$ & $\begin{array}{l}\text { Increases slowly } \\
\text { (Visceral obesity) }\end{array}$ & $\begin{array}{l}\text { Decreases rapidly } \\
\text { (Visceral obesity) }\end{array}$ & $\begin{array}{l}\text { Decreases slowly } \\
\text { (Unhealthy lean) }\end{array}$ \\
\hline Diabetes & Nondiabetic & \multicolumn{3}{|c|}{ Prediabetic } & Diabetic \\
\hline
\end{tabular}

The postprandial plasma glucose (PPG) elevates steadily with WBIR evolving, until the visceral adipose tissue as well as the subcutaneous adipose develops AIR in the IR-III stage and undergoes uninhibited lipolysis elevating plasma fatty acid (PFA) severely and thereby restricting plasma glucose (PG) utilization severely, and therefore it elevates steeply thereafter, until the liver develops HIR in the IR-IV stage undergoing unsuppressed hepatic glucose production (HGP), and therefore it elevates even more steeply thereafter; The postprandial plasma insulin (PPI) elevates steadily with PPG, until pancreatic insulin secretion starts to be impaired possibly around the end of IR-III stage, and therefore it saturates then decreases gradually thereafter; The fasting plasma insulin (FPI) would evolve similarly as PPI, but at a level attenuated significantly from PPI; The subcutaneous adipose de novo lipogenesis (s-ADNL) increases steadily, until the subcutaneous adipose tissue develops AIR in the IR-II stage, and therefore it decreases gradually thereafter; The visceral adipose de novo lipogenesis ( $v$-ADNL) increases steadily, until the visceral adipose tissue develops AIR in the IR-III stage, and therefore it decreases gradually thereafter; The postprandial plasma fatty acid (PPFA) declines gradually with PI, until the subcutaneous adipose tissue develops AIR in the IR-II stage undergoing uninhibited lipolysis, and therefore it elevates modestly thereafter, until even the visceral adipose tissue also develops AIR in the IR-III stage undergoing uninhibited lipolysis, and therefore it elevates steeply thereafter; The hepatic adipose de novo lipogenesis (HDNL) increases steadily with hepatic glucose uptake, until the liver develops HIR in the IR-IV stage, and therefore it decreases gradually thereafter; The fasting plasma triglyceride (FPTG) would elevates steadily with HDNL/VLDL generation, until the visceral adipose tissue as well as the subcutaneous adipose tissue develops AIR in the IR-III stage and fails to entrap PFAs hampering VLDL delipidation, and therefore it elevates steeply thereafter, until the liver develops HIR in the IR-IV stage, and therefore it declines gradually thereafter; The HGP would decrease gradually with PI, until the liver develops HIR in the IR-IV stage, and therefore it increases steeply; The FPG would decline gradually with PI, until the visceral adipose tissue as well as the subcutaneous adipose tissue develops AIR in the IR-III stage undergoing uninhibited lipolysis elevating PFA severely and thereby restricting PG utilization severely, and therefore it elevates 
gradually thereafter, until the liver undergoes unsuppressed HGP in the IR-IV stage, and therefore it elevates steeply; The HbA1c level would elevate steadily, until the visceral adipose tissue as well as the subcutaneous adipose tissue develops AIR in the IR-III stage and undergoes uninhibited lipolysis elevating PFA severely and thereby restricting PG utilization severely, and therefore it elevates steeply thereafter, until the liver develops HIR in the IR-IV stage undergoing unsuppressed HGP, and therefore it elevates even more steeply thereafter; The s-lipolysis is inhibited primarily by FPI and therefore decreases gradually, until the subcutaneous adipose tissue develops S-AIR in the IR-II stage, and therefore it increases gradually thereafter, until the subcutaneous fat mass is depleted enough, and therefore it saturates then decreases thereafter; The v-lipolysis is inhibited primarily by FPI and therefore decreases gradually, until the visceral adipose tissue develops AIR in the IR-III stage, and therefore it increases gradually thereafter, until the visceral fat mass is depleted enough, and therefore it saturates then decreases thereafter; The FPFA would decline gradually with FPI, until the subcutaneous adipose tissue develops AIR in the IR-II stage undergoing inhibited lipolysis, and therefore it elevates modestly thereafter, until even the visceral adipose develops AIR in the IR-III stage undergoing inhibited lipolysis, and therefore it increases steeply thereafter, until both the adipose tissues lose the fat mass enough, and therefore it saturates then decreases thereafter; The body weight increases rapidly in the IR-I stage, and then increase slowly in the IR-II stage, and then decreases rapidly in the IR-III stage, and then decrease slowly in the IR-IV stage; Lastly, $P P G_{o}, P P I_{o}$, $F P I_{o}, P P F A_{o}, F P T G_{o}, F P G_{o}, H b A c_{o}$, and FPFA denote reference values in the IS stage for PPG, PPI, FPI, PPFA, FPTG, FPG, HbA1c, and FPFA, respectively.

Actual measurement of WBIR-dependent metabolic evolution profiles would be extremely difficult, not only because WBIR evolution would generally proceed very slowly over years or even decades but also because detailed aspects of WBIR evolution would vary widely among individuals. Rather, WBIR-dependent metabolic evolution profiles may be drawn better heuristically, for a kind of standard individual, based on the descriptions in Table 3. Heuristically drawn WBIR-dependent evolution profile for PI, body weight, PFA, and PG is depicted in Fig 3. It is noted that heuristically drawn, rule of thumb profiles would be good enough as long as they provide general idea of how metabolic variables evolve from one stage to the next stage and at the same time are largely validated by the consistent experimental data in the integrated pool of resources described in Fig 2. 


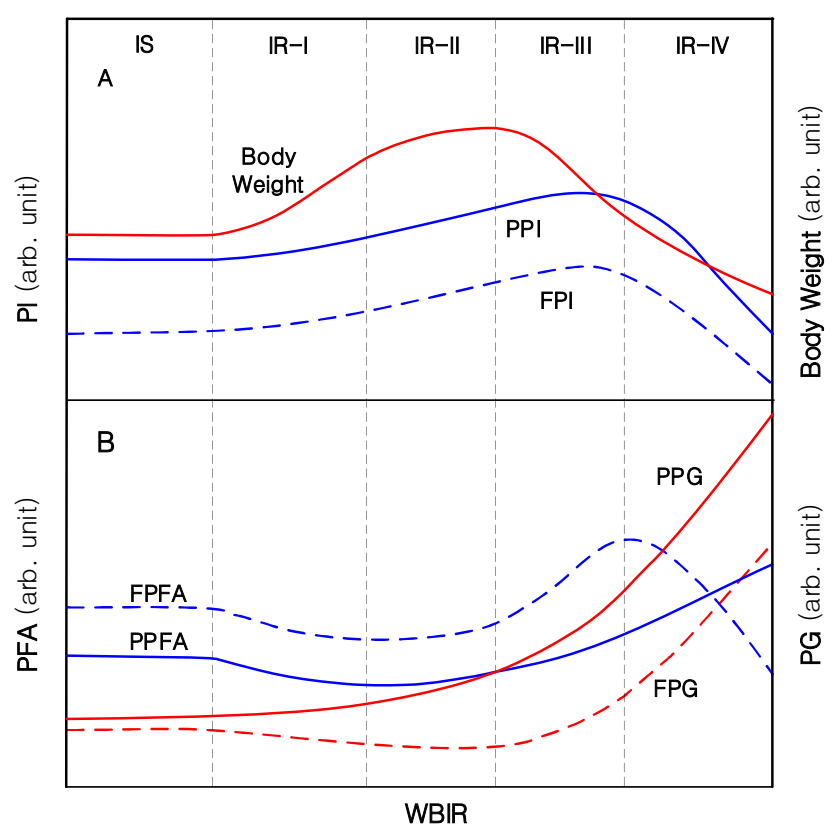

Fig 3. Heuristically drawn WBIR-dependent evolution profile for PI and body weight (A), and for PG and PFA (B), for a kind of standard individual.

Insulin is the major hormone that regulates the metabolism. This is why plasma insulin (PI) level is the most important determinant of the metabolism. As long as pancreatic insulin secretory response is largely intact as in the early stage of evolution, postprandial insulin secretion would be approximately proportional to postprandial plasma glucose (PPG), which would elevate steadily with WBIR evolving as more number of tissues or cells develop IR. Thus, post prandial insulin (PPI) would elevate steadily with WBIR evolving until insulin secretion starts to be impaired, possibly around the end of the IR-III stage, and then decline gradually thereafter. In general, the fasting plasma insulin (FPI) would drop significantly from PPI, as depicted in Fig 3(A), because PPI would decline gradually with time as the fed state gradually transitions to the fasted state.

First of all, WBIR-dependent evolution of PI would affect directly evolution of the body weight simply because insulin works in a pro-anabolic (2) as well as anti-lipolytic (36) manner. In the IR-I stage, for instance, MIR not only elevates PI but also redistributes some of the excess PG shunned from the MIR-affected muscle tissue to the adipose tissues $(37,38)$. Consequently, the elevated PPI would enhance fat accumulation in the fed state (pro-anabolic) by enhancing ADNL as well as FA entrapment from the plasma, whereas the elevated FPI would restrict lipolysis or fat mobilization in the fasted state (anti-lipolytic). Thus, both the AIR-free subcutaneous and visceral adipose tissues would increase the fat mass rapidly. In the IR-II stage, PI would be elevated further by s-AIR in addition to MIR. Consequently, the still AIR-free visceral adipose tissue would increase the fat mass even more rapidly while the AIR-affected subcutaneous tissue starts to lose the fat mass rapidly by undergoing uninhibited lipolysis, which eventually leads to visceral obesity with body weight still increasing overall. In the IR-III stage, even the visceral adipose tissue develops AIR and undergoes 
uninhibited lipolysis, leading to rapid weight loss. In the IR-IV stage, both the subcutaneous and visceral adipose tissue would eventually have lost most of the fat mass and then no longer be able to support lipolysis as effectively as in the IR-III stage, thereby slowing the pace of weight loss significantly. Overall, the body weight would evolve approximately in an inverted-U-shape with WBIR advancing as depicted in Fig 3(A): it would increase rapidly in the IR-I stage, then increase slowly in the IR-II stage, then decrease rapidly in the IR-III stage, and then decrease slowly in the IR-IV stage (9).

A noteworthy point in the body weight evolution profile in Fig $3(\mathrm{~A})$ is that the individuals belonging to the IR-I or IR-II stages - likely overweight or obese - would be relatively healthy metabolically simply because one or both of the subcutaneous and visceral adipose tissues work properly as a FA reservoir, thereby preventing PG as well as PFA from elevating too high. By contrast, the individuals belonging to the IR-III or IR-IV stages - likely relatively lean - would be rather unhealthy simply because both the adipose tissues fail to work effectively as a FA reservoir and therefore are unable to prevent PG as well as PFA from elevating severely. This would explain why some lean individuals have higher mortality than overweight or obese individuals - obesity paradox (13).

In fact, Lee et al. conducted a prospective cohort study, in which all-cause mortality had been traced during a follow-up period of 10.5 years, for subgroups of normoglycemia, impaired fasting glucose, newly diagnosed diabetes, and prevalent diabetes, classified at the baseline (39). And they found that the all-cause mortality varied, regardless of the baseline characteristics, approximately in a U-shape with BMI. More specifically, mortality increased much more steeply with BMI decreasing below the BMI having minimum mortality than it increased with $\mathrm{BMI}$ increasing above the $\mathrm{BMI}$ having minimum mortality. The mortality variation in a U-shape with BMI may conversely support our assertion that the body weight evolves approximately in an inverted-U shape with WBIR.

WBIR-dependent evolution of PI would also affect directly evolution of plasma fatty acid (PFA). In general, PFA level will depend on the intensity of adipose lipolysis and delipidation of triglyceride-rich lipoproteins (TRLs). In the fed state, the relatively high PPI would inhibit strongly adipose lipases so that the FA concentration inside the adipocytes plummets drastically. Consequently, in the fed state, the FAs in the plasma, including the ones released from TRLs, would diffuse back into adipocytes, thus lowering the postprandial plasma fatty acid (PPFA) significantly. In the fasted state, however, the relatively low FPI would inhibit adipose lipases less strongly so that the adipose tissues undergo lipolysis and release the resultant FAs into the plasma, thus elevating the fasting plasma fatty acid (FPFA) significantly. This explains why the PPFA is generally lower than the FPFA, as illustrated in the Fig 3(B). An important point here is that the PI level is higher in the IR-I stage than in the IS stage, which translates into that adipose lipases are inhibited more strongly in the IR-I stage than in the IS stage, which in turn translates into that PFA level is actually lower in the IR-I stage than in the IS stage, as illustrated in Fig 3(B). This also explains why insulin-sensitive healthy individuals, including most children, exhibit higher FPA level than insulin-resistant individuals 
(as in the IR-I or IR-II stage) who are more likely to be overweight or obese $(40,41)$. In the IR-II stage, the subcutaneous adipose tissue starts to develop AIR, not only releasing FAs into the plasma but also failing to entrap the FAs released from TRLs. However, the still largely AIR-free visceral adipose tissue would entrap, with the help of significantly elevated PI, most of the FAs shunned or released from the AIR-affected subcutaneous adipose tissue. Consequently, PFA elevation in the IR-II stage would be rather modest, as illustrated in the Fig 3(B). In the IR-III stage, however, both the subcutaneous and visceral adipose tissues are affected by AIR, and therefore they not only release FAs into the plasma but also fail to entrap the FAs released from TRLs, which elevates PFA severely. Most importantly, the severe elevation of PFA in the IR-III stage will inevitably restrict utilization of PG as competing energy substrate, which elevates PG significantly as well. In the IR-IV stage, both the subcutaneous and visceral adipose tissues would eventually have lost most of the fat mass and therefore no longer be able to support lipolysis as effectively as in the IR-III stage. Consequently, FPFA in the IR-IV stage would saturate and then decline gradually with WBIR, whereas PPFA would elevate steadily as both the adipose tissues are failing ever more to entrap the FAs released from TRLs. This would explain why both the FIRKO and A-ZIP/F-1 mice, lacking adipose tissues working effectively working as a FA reservoir, exhibit PPFA level actually higher than FPFA level $(5,27)$.

WBIR-dependent evolution of PI would also affect directly evolution of PG, but quite differently for the postprandial plasma glucose (PPG) and fasting plasma glucose (FPG). PG in general has two distinct components: the exogenous PG attributed to ingested carbohydrates and the endogenous PG attributed to hepatic glucose production (HGP). In the fed state, however, HGP would be suppressed enough by relatively high PPI, as long as the liver is largely free from HIR as in the early stage of WBIR evolution. Consequently, the exogenous PG in general would be disposed of rather rapidly within the fed state - especially with the help of significantly elevated PPI - by the tissues still largely IR-free. This implies that in the early stage of evolution FPG would be determined primarily by HGP, which is regulated primarily by FPI - the higher FPI, the more suppressed the HGP, the more lowering FPG. Obviously, higher FPI would be attributed to higher PPI, which would in turn be attributed to higher PPG. This line of reasoning would be reduced to: in the case of the early stage of evolution, FPG would decline with PPG elevating, as illustrated in Fig 3(B). However, the negative correlation between FPG and PPG would eventually turn positive in the IR-III stage or in the IR-IV stage as the severely elevated PFAs restrict PG utilization or HIR makes the liver undergo unsuppressed HGP, respectively, thereby elevating not only PPG but also FPG severely.

In fact, Hulman et al. traced the glycemic evolution up to diagnosis of type 2 diabetes in the Whitehall prospective cohort study (42). We reproduced in Fig 4 the evolution profiles of $2 \mathrm{hPG}$ (2-hour plasma glucose) measured by oral glucose tolerance test (OGTT) and FPG for incident diabetics who had been diagnosed of diabetes during the follow-up from 1991 to 2013. The 2hPG, which would represent approximately the PPG, elevated steadily with time before diagnosis of diabetes, whereas FPG actually declined with time, only to elevate steeply near the diagnosis. It is also notable that far from the diagnosis, the South Asian subgroup having higher FPI exhibited FPG 
lower than in the white subgroup having lower FPI. An important point here is that the horizontal axis of the time before diagnosis would not necessarily correspond to the WBIR evolution stage introduced in this study, given that individuals would develop diabetes with widely different paces, depending on the concentration of potential IR-inducing agents. In general, the farther way from the diagnosis, the more heterogeneous the subjects in terms of WBIR evolution stage.
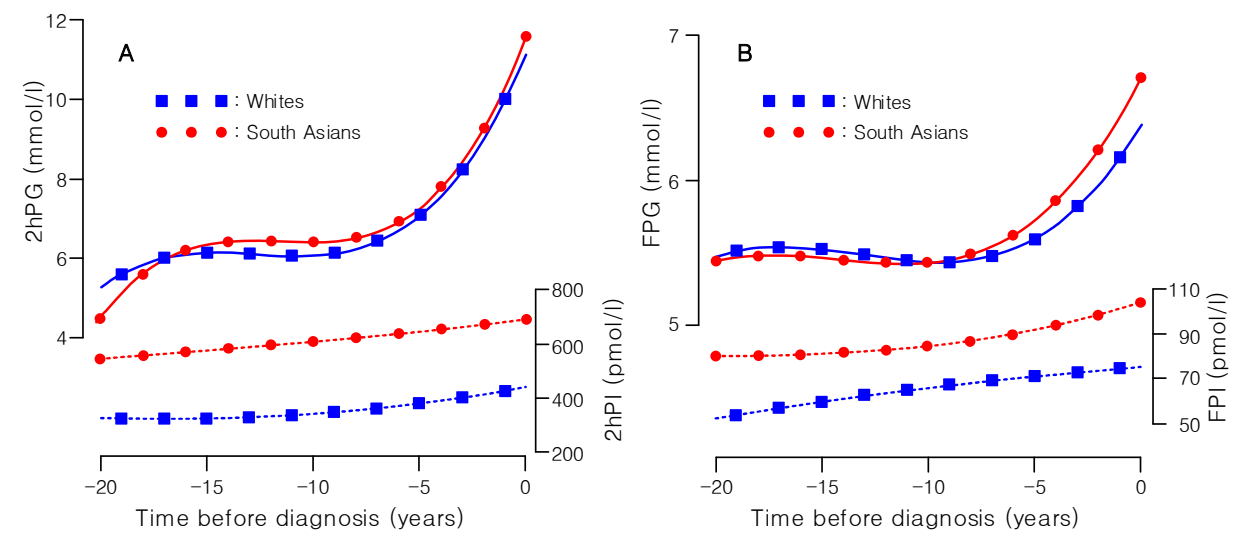

Fig 4 Variation of $2 \mathrm{hPG}$ and $2 \mathrm{hPI}(\mathrm{A})$, and FPG and FPI (B) before diagnosis of diabetes for the white subgroup and South Asian subgroup

Actually earlier, Tabak et al. had also traced glycemic evolution with the same Whitehall cohort study (43). However, they divided the subjects into the subgroup of incident diabetics who had been diagnosed of diabetes during the follow-up and the subgroup who had remained nondiabetic, and then plotted glycemic evolution against the time before the end of follow-up, rather than the time before the diagnosis. While the FPG in the nondiabetic subgroup remained almost constant throughout the follow-up, the FPG in the incident diabetics elevated at a relatively low rate in the region far from the end of follow-up and then suddenly elevated steeply near the end of follow-up. The positive slope of the FPG with time before the end of the follow-up - in contrast to the negative slope with time before diagnosis - would indicate that subjects in the horizontal axis of the time before the end of follow-up were much more heterogeneous in terms of WBIR evolution stage.

On the other hand, Yeni-Komshian et al. conducted an insulin suppression test with nondiabetic individuals (possibly belonging to the IR-I and IR-II stages), in which steady-state plasma glucose (SSPG) concentration is measured under continuous infusion of insulin and glucose (44). And they found that FPG was negatively correlated with the SSPG, which would also represent approximately PPG. This also confirms that the FPG actually declines with PPG in the individuals who are nondiabetic and thus are more likely in the early stages of WBIR evolution.

As described above, FPG would tend to remain relatively low until very late in the course of diabetes development (45). An important point here is that measurement of FPG for the purpose of diagnosing diabetes would be more likely to give false sense of security when in fact WBIR has advanced already significantly, which would make it more difficult to detect diabetes early. 
A noteworthy point regarding the evolution profiles of FPFA and FPG in Fig 3(B) may be that both the FPFA and FPG (the primary and secondary fasting energy substrate, respectively) are actually lower in the weight gaining IR-I and IR-II stages than in the IS stage. The lack of the fasting energy substrates in the plasma would be more likely to enhance the hunger felt in the fasted state (46) likely leading to overeating - as well as discourage physical activity. The likelihood of overeating and physical inactiveness would easily lead to positive energy balance essential for weight gaining.

The four-stage evolution of diabetes from rapid weight gain to visceral obesity to rapid weight loss to full-blown diabetic state seems to comply rather well with the natural development history of diabetes (1). This may also conversely validate the four-stage WBIR evolution model. In fact, the validity of the four-stage WBIR evolution model would rest primarily on the validity of the postulation that tissue-specific IRs develop in the order of MIR, s-AIR, V-AIR, and HIR. A simple way to check the validity may be to assume a different order of tissue-specific IR development and compare the predicted metabolic outcomes with the familiar observations. For instance, if AIR develop first - as is the case with the FIRKO mice - WBIR evolution would start with rapid weight loss, rather than rapid weight gain. And if HIR develop first - as is the case with the LIRKO mice fasting hyperglycemia would be observed from the early stage of WBIR evolution. And if v-AIR precede s-AIR, visceral obesity would never be observed. And if all the MIR, S-AIR, V-AIR, and HIR develop simultaneously, FPG would elevate steadily as PPG, and FPFA would elevate steadily as PPFA, whereas body weight would decrease steadily. An interesting point is that, in type 1 diabetics with no or little insulin secretion, insulin action would be missing in all of the tissues simultaneously, making the metabolic disruptions due to MIR, s-AIR, v-AIR, and HIR occur almost simultaneously. This may be why untreated type 1 diabetics become emaciated rapidly, possibly without showing any sign of visceral obesity.

If the four-stage WBIR evolution model is indeed valid, there would exist some surrogate measures that represent the degree of WBIR evolution (44) fairly well. A good candidate would be the PPG that elevates steadily with WBIR evolution $(45,47,48)$ - PPG reflects all of MIR, s-AIR, v-AIR, and $H I R$. On the other hand, the FPG would be an utterly poor surrogate measure simply because it declines with WBIR in the early stage of evolution. Both the PPI and FPI would not be a good surrogate measure, either - they tend to saturate or decline in the later stage of WBIR evolution (44). The FPFA would not be a good surrogate measure, either - it dips low in the IR-I and IR-II stages and saturates or declines with fat mass depleted enough in the IR-IV stage. The fasting plasma triglyceride (FPTG) would not be a good surrogate measure, either - it declines in the IR-IV stage. Body weight or BMI would be an utterly poor surrogate measure - it evolves approximately in an inverted-U-shape with WBIR advancing. Probably, the best surrogate measure may be the $\mathrm{HbA1C}$ level, which is known to reflect the time-averaged PG in the preceding 10-12 weeks and therefore takes into account both the fasting glycemia and the postprandial glycemia (48). 


\section{Debunking of the lipid-induced insulin resistance (LIIR)}

Even though the four-stage WBIR evolution model is largely validated, it does not tell anything about IR-inducing agents or mechanisms. This means that the model would be complete only if it is coupled with a proper IR-inducing mechanism. A widespread belief is that IR is induced by lipids. A core argument of the so-called lipid-induced insulin resistance (LIIR) is that fat mass or obesity induces IR either directly, or indirectly by contributing excess FAs to the plasma so that the resultant elevated PFAs somehow interfere the insulin action in cells or tissues (11)

In fact, Karpe et al. extensively reviewed literatures only to find that the correlation between the body weight and PFA is not very straightforward (11). For instance, some obese or overweight individuals exhibited PFA actually lower than the average, whereas lean individuals exhibited a very wide range of PFA from below to well above the average - actually complying well with the heuristically drawn PFA evolution profile in Fig 3(B). Yet elevation of PFA still appears to apparently impair glucose tolerance or elevate $P G$, but only because it tends to restrict utilization of competing energy substrate PG - Randle cycle or glucose-FA cycle $(28,29)$. An important point is that the more insulin-resistant, the more handicapped the PG in competing with PFA for energy substrates - PG requiring insulin mediation will have serious difficulty in transporting into insulin-resistant cells, whereas PFAs will diffuse rather freely into cells regardless of the IR status of tissues $(24,25)$. This may be why PFAs are preferentially utilized over PG in severe diabetics with both PG and PFA simultaneously elevated severely (31). At the same time, this may also explain why elevation of PFA looks as if to impair glucose tolerance more severely in diabetics, apparently lending support to the LIIR.

However, a crucially important point here is that severe elevation of PFA is in reality a hallmark of $A I R$, as can be seen in Table 1. If elevation of PFA indeed has a property to induce IR, insulin-sensitive individuals as in the insulin-sensitive (IS) stage - more likely lean and having relatively high PFA would be more susceptible to IR than already insulin-resistant individuals as in the IR-I or IR-II stage - more likely overweight or obese and having relatively low PFA. This is obviously an absurdity. An important point here is that weight gaining is in itself an indication that adipose tissues are AIR-free and properly containing FAs in the form of fats, thereby preventing PFA from elevating too high. This refutes flatly the LIIR.

An obvious problem with the LIIR is that it does not tell which tissue-specific IR is induced by obesity or lipids. From the perspective of WBIR evolution model, however, MIR in the IR-I stage contributes to weight gain or obesity - not the other way around - by not only elevating PI but also effectively redistributing some of the excess PG shunned from the MIR-affected muscle tissue to adipose tissues, whereas AIR, for instance, in the IR-III stage incurs rapid weight loss by making adipose tissues undergo uninhibited lipolysis. This also largely refutes the LIIR.

Another core argument of the LIIR is that ectopic fats induce IR. For instance, many researchers believe that HIR is attributed to the ectopic fats deposited in the liver $(49,50)$. However, basics of 
physiology and metabolism suggests that enhanced ectopic liver fat deposition is more likely an effect AIR in particular. First of all, AIR elevates severely PFAs by making the adipose tissues undergo uninhibited lipolysis and fail to entrap the FAs released from triglyceride-rich lipoproteins (TRLS) severe elevation of PFA is a hallmark of AIR. The severely elevated PFAs, shunned, in fact, by the AIR-affected adipose tissues, would have no choice but to diffuse also into the liver and esterify into ectopic liver fat (26). A supporting evidence is that the FIRKO and A-ZIP/F-1 mice, both lacking the adipose tissue working properly as FA reservoir and therefore exhibiting almost the same phenotype, deposit significant amount of ectopic liver fat $(5,27)$. Ectopic liver fat may also be deposited via enhanced hepatic de novo lipogenesis (HDNL) following enhanced hepatic glucose uptake (50). However, enhancement of HDNL may also be attributed to AIR. In more detail, AIR elevates not only PFA but also PG, thereby enhancing hepatic glucose uptake and HDNL only if the hepatic insulin signaling is largely intact or the liver is not yet affected by HIR - in fact, enhanced HDNL as well as enhanced hepatic glucose uptake is in itself an indication that liver is still largely free from HIR. The enhanced HDNL would in turn enhance VLDL generation and its secretion to the plasma. However, the severely elevated PFAs due to AIR would hamper VLDL delipidation (22), which would in turn hamper VLDL secretion to the plasma, which would in turn inevitably contribute to accumulation of ectopic liver fat. The reasoning above leads to the conclusion: increased ectopic liver fat deposition via either enhanced HDNL or enhanced hepatic influx of FAs has more to do with AIR, rather than with HIR. A noteworthy point here is that a hallmark of HIR is unsuppressed HGP or fasting hyperglycemia, rather than enhanced ectopic fat deposition.

A serious dilemma with the notion that ectopic fats induce IR is that even healthy individuals, who are more likely free from IR in general, also accumulate a considerable amount of intramyocelluar lipid (IMCL) (51) - ectopic fat deposited in muscle tissues - especially when they fast (52), engage in prolonged exercise (53), or rely on low-carbohydrate diet (LCD) (54). Fasting would lower the FPI significantly, which enhances lipolysis significantly, thereby elevating PFA significantly and subsequently increasing FA flux into muscle tissues as well to increase IMCL deposition. Prolonged exercise would also elevate PFAs significantly, eventually increasing IMCL deposition. LCD would decrease significantly the postprandial insulin secretion and lower FPI significantly, which enhances lipolysis significantly, enhancing significantly PFA and eventually enhancing IMCL deposition.

The observation that even healthy individuals deposit a considerable amount of ectopic fats may suggest that ectopic fat deposition is in reality a kind of natural homeostatic response of the body to prevent severe elevation of PFA. In order to achieve PFA homeostasis more efficiently, ectopic fats deposited usually in the fasted state should be oxidized as early as possible in the following fed state, which would inevitably delay glucose oxidation and thus apparently elevate PG significantly especially in the postprandial period.

Kanamori et al. conducted an interesting experiment, in which healthy subjects were fed on high-carbohydrate diet (HCD) on day 1 (D1) and D2, and switched to low-carbohydrate diet (LCD) 
on D3, and switched back to the same HCD on D4 (55). Interestingly, PPG was elevated much higher on D4 than on D2, even though the subjects ingested the same HCD on the two days. Proponents of LIIR may argue that the apparent impairment of glucose tolerance (or elevation of PPG) on D4 serves as evidence that the elevated PFA or increased ectopic fat deposition on D3 (due to LCD) induces IR. However, given that IR is a long-term phenomenon that generally develops over a long span of time, the apparent impairment of glucose tolerance after having LCD for only a day can hardly be attributable to IR development. If IR were induced simply by a change of diet for only a day, it could be reversed easily by correcting diet for only a few days. Similarly, the apparent impairment of glucose tolerance after exercise or fasting can hardly be attributable to IR development, considering especially that fasting had once been prescribed as an important option for treating diabetes (56) and exercise is still widely practiced for managing diabetes.

\section{Glycation-induced insulin resistance (GIIR) proposed}

Now that the LIIR is largely debunked (especially by the four-stage WBIR evolution model), it would be essential to introduce an appropriate IR-inducing mechanism that complies well with the four-stage WBIR evolution model. An important aspect of WBIR evolution is that PG or, more accurately, time-averaged PG elevates at an ever accelerated pace with WBIR evolving, as described in Table 3. A speculation is that hyperglycemia may have an inherent property to induce or worsen IR. For instance, even type 1 diabetics, who have little or no insulin secretion and therefore are inevitably exposed to severe hyperglycemia, also develop severe IR $(57,58)$. The A-ZIP/F-1 mice, lacking adipose tissues to work as a FA reservoir and therefore inevitably exposed to severe elevation of PG as well as PFA, eventually develop diabetes, but surgical implantation of wild-type adipose tissue not only normalizes PG but also reverses the diabetes with improved insulin sensitivity as well (59). Furthermore, most diabetes management or treatment plans, including exercise and administration of anti-diabetic medications (ADMs), make a point of lowering PG.

A reaction activated especially in hyperglycemic physiological condition is glycation or non-enzymatic glycosylation, in which glucose and its glycolytic derivatives, such as glyoxal and methylglyoxal, react non-enzymatically with proteins and form covalently bonded adducts $(14,15)$. If hyperglycemia is sustained long enough, the glycation adducts would rearrange themselves into nonreversible advanced glycation end-products (AGEs).

An important aspect of the glycation reaction is that glycation adducts are formed preferentially on the positively charged (of either polarized or ionic) constituents of proteins, such as $\mathrm{N}$-terminus amino group and residues of lysine, arginine, and cysteine. Most importantly, glycation adducts may effectively neutralize the positive charges on the glycated residues (16). In this case, the attractive forces between the positively charged residues glycated and the nearby negatively charged residues will disappear, possibly making the polypeptide chain unfold locally. An important point is that the proteins with both the charge distribution and conformation disrupted significantly can hardly function properly. 
Another important aspect of the protein glycation is that cellular proteins in general are glycated more readily than extracellular proteins in the plasma or the interstitial fluid (60). Plasma membrane proteins on the surface of the cells in tissues, certainly a category of cellular proteins, may particularly be susceptible to glycation, simply because they are exposed not only to glucose in the interstitial fluid but also to the very potent glycating agents, such as glyoxal and methylglyoxal that may be produced primarily inside the cells as glycolytic byproducts (61).

An example of the plasma membrane proteins is insulin receptors that mediate insulin action in cells. If insulin receptors are glycated enough such that their charge distribution and conformation are hardly compatible with those of insulin, insulin will not be able to bind properly to glycated insulin receptors, to say nothing of initiation of insulin signaling. The failure of insulin to initiate insulin signaling, as in the mice with insulin receptors knocked-out (3-5), as a result of glycation of insulin receptors or other proteins directly involved in downstream insulin signaling would lead to nothing but the glycation-induced insulin resistance (GIIR). Actually, the authors had briefly discussed this IR-causation mechanism elsewhere (62).

An important point is that glycation reaction is a kind of a long-term process that takes place rather slowly over a relatively long span of time, exactly like the WBIR evolution. In contrast, the glucose-FA cycle is a short-term process that responds quickly to the variation of PFA concentration (51). This may possibly support that WBIR evolution indeed involves glycation reaction. Another revealing observation is that an anti-diabetic medication metformin - probably the most frequently prescribed oral hypoglycemic agent $(\mathrm{OHA})$ - is known to inhibit glycation process. This may also suggest conversely that IR development or WBIR evolution indeed involves glycation reaction (6365).

If IR is indeed induced by glycation reaction activated in hyperglycemia, the resultant GIIR will impair glucose uptake further, which will in turn enhance hyperglycemia and GIIR further, inevitably establishing a positive feedback loop in which hyperglycemia and GIIR reinforce each other in a vicious circle. As discussed already, the four-stage WBIR evolution model indeed predicts that PG or time-averaged PG elevates at an ever faster pace with WBIR evolving. The good reconciliation of the four-stage WBIR evolution model with GIIR may conversely suggest that GIIR is the very IR-inducing mechanism behind WBIR evolution. The most important implication of GIIR underlying the WBIR evolution is that hyperglycemia is not only the effect but also the main driving force for WBIR evolution. The four-stage WBIR evolution with GIIR as the IR-inducing mechanism can be described as below.

Hyperglycemia, the main driving force for WBIR evolution, can easily be achieved, at least in the postprandial period, even in the insulin-sensitive (IS) stage by increasing postprandial glycemic load or increasing carbohydrate intake. With postprandial hyperglycemia sustained for long enough a period, for instance, by relying on high-carbohydrate diet (HCD), GIIR would be more likely to develop gradually first preferentially in the muscle tissue, in which the relatively low cell turnover 
would make the average cells be exposed longer to hyperglycemia. With the muscle tissue gradually developing IR (more specifically MIR), the IS stage would gradually transition to IR-I stage. Meanwhile, MIR in the IR-I stage would then impair the muscle glucose uptake and enhance postprandial hyperglycemia further, which would subsequently enhance postprandial insulin secretion, leading to hyperinsulinemia. Hyperinsulinemia in concert with postprandial hyperglycemia would then gradually increase the body weight to the level expected in the IR-I stage. On the other hand, the hyperglycemia enhanced further by MIR would eventually make even the subcutaneous adipose tissue develop gradually GIIR (more specifically s-AIR). With s-AIR developing gradually, the IR-I stage would gradually transition to the IR-II stage, in which MIR and S-AIR would enhance hyperglycemia further by impairing glucose uptake in the muscle tissues and subcutaneous adipose tissue, respectively. Thus, even the visceral adipose tissue would develop gradually GIIR (more specifically v-AIR). With v-AIR developing gradually, the IR-II stage would gradually transition to the IR-III stage, in which severe elevation of PFA would enhance hyperglycemia even more severely by restricting PG utilization. Thus, even the liver with a very high cell turnover would develop gradually GIIR (more specifically HIR). With HIR developing gradually, the IR-III stage would gradually transition to IR-IV stage, in which HIR would make the liver undergo unsuppressed HGP, enhancing hyperglycemia even more.

An indisputable evidence for active glycation reaction in hyperglycemia will be a disproportionately high plasma level of glycation free adducts - glycated amino acids or glycated peptides released from the glycated proteins undergoing proteolysis - observed in diabetics (66, 67). Glycation free adducts with a relatively small size will be released first into the interstitial fluid bathing all the cells in tissues and then eventually be collected in the blood plasma. On the other hand, the glycation adduct residues still covalently-bonded to the proteins constituting the tissues can rarely be detected in the plasma. Nevertheless, the glycation adduct residues may be extremely harmful or toxic simply because they could disable the host proteins in cells or tissues. By contrast, glycation free adducts may be considered only a risk marker, rather than a risk factor, at least because they no longer affect directly the function of the cells or tissues and at the same time they are mostly excreted in urine $(66,67)$.

A speculation is that glycation reaction may possibly act as a common thread connecting together various disorders associated with diabetes. For instance, MIR, s-AIR, v-AIR, and HIR may be considered to be associated with each other simply because they are induced - in the perspective of GIIR - by glycation of the proteins involved in insulin action in the muscle tissue, subcutaneous adipose tissue, visceral adipose tissue, and liver, respectively. An important point here is that even though MIR, s-AIR, v-AIR, and HIR are associated with each other, they will not necessarily develop simultaneously. If the proteins involved in insulin action in peripheral tissues were glycated severely in hyperglycemia, some proteins involved in insulin generation in pancreas might also be glycated such that insulin generation is severely impaired. This may explain why impairment of insulin action in tissues (or tissue-specific IR) is associated with the impairment of insulin secretion in pancreas. In 
fact, it was found that chronic hyperglycemia triggers loss of pancreatic beta cell differentiation (68), possibly supporting that glycation reaction is also involved in impairment of insulin secretion. To take the argument one step further, if the proteins involved in insulin action and secretion in different tissues or organs were glycated severely in hyperglycemia, some critical proteins in still other tissues or organs, such as cardio/cerebral endothelia, nephrons, neurons, and retina could also be glycated severely such that those tissues or organs are deteriorated functionally or structurally as to exhibit cardio/cerebral vasculopathy, nephropathy, neuropathy, and retinopathy, respectively. This may possibly explain why various diabetes-associated disorders or diseases are associated with each other.

Perhaps one of the best known examples of glycated protein may be the glycated version of hemoglobin, often referred to as $\mathrm{HbA} 1 \mathrm{c}$, which is formed inside the red blood cells during the life span of three to four months (69). As well known, the level of $\mathrm{HbA} 1 \mathrm{c}$ represents the average plasma glucose concentration in the preceding 10-12 weeks. From the perspective of GIIR, glycation reaction is the main driving force for WBIR evolution, and it is certainly the main driving force for the formation of glycated hemoglobin HbA1c. Then, it would hardly be incidental that the HbA1c level is one of the best surrogate measures of WBIR evolution, as predicted in this study.

Lastly, GIIR would also hold for type 1 diabetes $(57,58)$, in which the hyperglycemia that induces GIIR is attributable to lack of insulin secretion. If typ1 diabetes were left untreated, metabolic disruptions due to MIR, s-AIR, v-AIR, and HIR occur almost simultaneously, elevating PG very rapidly and thereby accelerating GIIR drastically. This means in other words that delay of insulin injection therapy in type 1 diabetes would accelerate WBIR evolution rapidly, only to increase the dose of insulin required.

\section{Discussion}

We have developed the four-stage WBIR or diabetes evolution model based on integrated approach that we have newly introduced. The model not only resolves most of the controversies on obesity or diabetes, including visceral obesity, obesity paradox, and dawn phenomenon that have long remained a puzzle, but also provides valuable insights on the true nature of the association between obesity and diabetes.

The single most important conclusion in this study is that hyperglycemia is the primal risk factor or main driving force for the WBIR evolution or diabetes development. Then, the most obvious approach to prevent, delay, or reverse WBIR evolution or diabetes development is to alleviate hyperglycemia, which is in fact the primary goal of diabetes management of treatment. And an obvious approach to achieve alleviation of hyperglycemia is to reduce postprandial glycemic load by curtailing carbohydrate intake.

One of the most important findings in this study is that weight gaining or obesity has an effect of alleviating hyperglycemia, the main driving force for WBIR evolution. This means in other words 
that weight gaining or obesity would in reality have an effect of delaying WBIR evolution or diabetes development - far from inducing IR or causing diabetes. This in turn means that weight gaining or obesity would hardly be a risk factor per se. In fact, it was known that even the visceral obesity has protective effect (70).

Even though weight gaining or obesity is not a risk factor per se (because it has an effect of alleviating hyperglycemia, the primal risk factor), it would hardly be beneficial overall for health. This suggests strongly that behind the weight gaining or obesity lies an independent risk factor, which is nothing but the hyperglycemia itself - it is not proper to find fault with weight gaining or obesity, rather than with hyperglycemia. This means in other words that hyperglycemia is also a risk factor or a necessary condition for weight gaining - hyperglycemia promotes weight gain only prior to development of AIR or impairment of pancreatic insulin secretion. Then, an obvious approach to achieve healthy weight loss would be to alleviate hyperglycemia.

The rapid weight gaining in the IR-I stage, for instance, may easily be prevented or reversed by alleviating hyperglycemia by reducing postprandial glycemic load or curtailing carbohydrate intake. The alleviation of postprandial hyperglycemia would decrease postprandial insulin secretion, which subsequently leads to alleviation of hyperinsulinemia. The alleviation of hyperinsulinemia would restrict, in concert with the alleviation of postprandial hyperglycemia, fat accumulation in the fed state and enhance more or less lipolysis or fat mobilization in the fasted state, thereby contributing to weight loss. Furthermore, the lipolysis and HGP more or less enhanced by alleviated hyperinsulinemia would elevate significantly FPFA and FPG, respectively. Relative abundance of the fasting energy substrates of FPFA and FPG in the plasma would be more likely to relax the hunger felt in the fasted state $(46,71)$ - likely leading to moderate eating - as well as encourage physical activity. The likelihood of moderate eating and physical activeness would easily lead to negative energy balance essential for weight loss.

Alleviating hyperglycemia in the IR-I stage for long enough a period as to reduce the body weight to the level expected in the IS stage may also reverse WBIR from the IR-I stage to the IS stage. This would be in line with our earlier assertion that postprandial hyperglycemia retained for long enough a period in the IS stage, for instance, by relying on high-carbohydrate diet (HCD) would not only advance WBIR to the IR-I stage but also increase the body weight to the level expected in the IR-I stage. An important point here is that the weight loss achieved by alleviating hyperglycemia would be of a healthy kind since it helps to secure more of the storage capacity of the FA reservoir. By contrast, the weight loss associated with AIR, as observed in the IR-III or IR-IV stage, is of an unhealthy kind since it shrinks further the storage capacity of the FA reservoir and therefore effectively enhances hyperglycemia further.

A notable aspect of this study may be that we have employed a new methodology named integrated approach. The methodology relies on integrated pool of resources consisting of the resource of basics and the resource of wide range of consistent experimental data obtained from 
across the wide range of disciplines. Thus, the theories or conclusions drawn based on integrated approach would generally be much more reliable than those obtained by simple experimental studies that largely ignores the basics of physiology or metabolism. Most importantly, the reliable theories or conclusions achieved by integrated approach would help rectify the misconceptions or cause-effect confusions frequently observed in medical researches.

One of the most persistent misconceptions regarding obesity or diabetes may be that weight gaining or obesity induces IR or causes diabetes. This misconception may possibly be attributed to the familiar observation that diabetes development starts with rapid weight gaining in the early stage of evolution, even though newly diagnosed diabetics lose weight rapidly only to gain some weight back only if treated by insulin or oral hypoglycemic agents (OHAs). The persistence of the misconception may possibly suggest conversely that the conventional methodology to conduct researches especially on obesity or diabetes has serious limitations.

A prevailing trend in current researches on obesity or diabetes may be that they are mostly done by simple experiments that largely ignore basics of physiology/metabolism and often measure mainly the association, for instance, between the body weight and IR - not tissue-specific IR. However, association alone in general does not tell much about the causality between associated variables and often leads to the fallacy of assuming the association as causality. For example, simple measurement of the association between weight gaining and IR - not tissue-specific IRs - in the early stage of evolution would easily lead one to argue falsely that weight gaining induces IR. It is noted, however, that the association between weight gaining and IR in the early stage of evolution tells nothing but that they have the common risk factor of hyperglycemia. A noteworthy point here is that it may not be a simple task to measure tissue-specific IRs (72). For example, the gold standard techniques established for measuring insulin sensitivity/resistance, such as hyperinsulinemic euglycemic clamp (HEC) and oral glucose tolerance test (OGTT), measure only average values in the whole body, rather than tissue-specific values.

Another important finding in this study is that the body weight or body mass index (BMI) is an utterly poor surrogate measure of unhealthiness of individuals, simply because body weight evolves approximately in an inverted-U shape with WBIR advancing. This would be why research after research had found that the mortality vs BMI curve has approximately a U-shape or J-shape (39, 73-76), which confirms nothing but the obesity paradox.

Notwithstanding the obesity paradox, however, many researchers may still be obsessed with the notion that weight gaining or obesity per se is a serious risk factor. This obsession might make some researchers be biased in interpreting their experimental results. For instance, researchers, probably from the same research institute, had made conflicting interpretations of the AIR model, FIRKO mice in studies reported in 2002, 2003, and 2016. More specifically, the 2002 study insisted that FIRKO mice were protected against obesity and obesity-related glucose intolerance (77), and the 2003 study insisted that the mice could extend longevity (78), whereas the 2016 study found 
that the mice developed diabetes as well as nonalcoholic fatty liver disease (NFLD) (5). According to our understanding, however, the AIR in the FIRKO mice is a very lethal disorder that elevates severely PG as well as PFA and eventually makes the mice develop diabetes as well as NFLD. Thus, the interpretation in the 2016 study is much closer to the truth, which is basically why we referred only the 2016 study and, at the same time, we could not incorporate the arguments made in the 2002 and 2003 studies into the integrated pool of resources crucial for integrated approach.

Lastly, we believe that the integrated approach that requires continual survey of literatures for replenishment or refinement of integrated pool of resources would help expedite sharing of important findings across the wide range of disciplines. For instance, if the finding in 2000 that surgical implantation of wild-type adipose tissue into the A-ZIP/F-1 mice exhibiting almost the same phenotype as the FIRKO mice not only normalizes PG but also reverses the diabetes (59) were shared across the wide range of disciplines, the misconception that weight gaining or obesity causes diabetes - or that lipids induces IR - may no longer prevail. On the other hand, such claims that FIRKO mice exhibiting almost the same phenotype as the A-ZIP/F-1 mice were protected against the obesity-related glucose intolerance (77) or could extend longevity (78) would not have been made in the first place.

\section{Acknowledgement}

The authors appreciate the support of Chungnam National University for this study.

Data Availability Statement: No data have been produced by this study itself.

Funding: The authors received no specific funding for this work.

1. Weyer C, Bogardus C, Mott DM, Pratley RE. The natural history of insulin secretory dysfunction and insulin resistance in the pathogenesis of type 2 diabetes mellitus. J Clin Invest. 1999;104(6):787-94.

2. Dimitriadis G, Mitrou $P$, Lambadiari $V$, Maratou E, Raptis SA. Insulin effects in muscle and adipose tissue. Diabetes Res Clin Pract. 2011;93 Suppl 1:S52-9.

3. Bruning JC, Michael MD, Winnay JN, Hayashi T, Horsch D, Accili D, et al. A muscle-specific insulin receptor knockout exhibits features of the metabolic syndrome of NIDDM without altering glucose tolerance. Mol Cell. 1998;2(5):559-69.

4. Michael MD, Kulkarni RN, Postic C, Previs SF, Shulman GI, Magnuson MA, et al. Loss of insulin signaling in hepatocytes leads to severe insulin resistance and progressive hepatic dysfunction. Mol Cell. 2000;6(1):87-97.

5. Softic S, Boucher J, Solheim MH, Fujisaka S, Haering MF, Homan EP, et al. Lipodystrophy Due to Adipose Tissue-Specific Insulin Receptor Knockout Results in Progressive NAFLD. Diabetes. 2016;65(8):2187-200.

6. Rosen ED, MacDougald OA. Adipocyte differentiation from the inside out. Nat Rev Mol Cell 
Biol. 2006;7(12):885-96.

7. Milo R, Philips, R. Cell Biology by the numbers. New York: Garland Science; 2016.

8. Kim SM, Lun M, Wang M, Senyo SE, Guillermier C, Patwari $P$, et al. Loss of white adipose hyperplastic potential is associated with enhanced susceptibility to insulin resistance. Cell Metab. 2014;20(6):1049-58.

9. Lee SJ, Kim,W., Shin,S.W. Whole-body insulin resistance (WBIR) evolving in four stages and its evolutionary effect on the body weight. ECE 2019; 18-21 May 2019; Lyon, Fracne2019.

10. Saad MF, Knowler WC, Pettitt DJ, Nelson RG, Charles MA, Bennett PH. A two-step model for development of non-insulin-dependent diabetes. Am J Med. 1991;90(2):229-35.

11. Karpe F, Dickmann JR, Frayn KN. Fatty acids, obesity, and insulin resistance: time for a reevaluation. Diabetes. 2011;60(10):2441-9.

12. McFarlane SI. Antidiabetic medications and weight gain: implications for the practicing physician. Curr Diab Rep. 2009;9(3):249-54.

13. Hainer V, Aldhoon-Hainerova I. Obesity paradox does exist. Diabetes Care. 2013;36 Suppl 2:S276-81.

14. Rabbani N, Thornalley PJ. Dicarbonyls linked to damage in the powerhouse: glycation of mitochondrial proteins and oxidative stress. Biochem Soc Trans. 2008;36(Pt 5):1045-50.

15. Zhang Q, Ames JM, Smith RD, Baynes JW, Metz TO. A perspective on the Maillard reaction and the analysis of protein glycation by mass spectrometry: probing the pathogenesis of chronic disease. J Proteome Res. 2009;8(2):754-69.

16. Pun PB, Murphy MP. Pathological significance of mitochondrial glycation. Int J Cell Biol. 2012;2012:843505.

17. Richter EA, Hargreaves M. Exercise, GLUT4, and skeletal muscle glucose uptake. Physiol Rev. 2013;93(3):993-1017.

18. Slimani L, Oikonen V, Hallsten K, Savisto N, Knuuti J, Nuutila P, et al. Exercise restores skeletal muscle glucose delivery but not insulin-mediated glucose transport and phosphorylation in obese subjects. J Clin Endocrinol Metab. 2006;91(9):3394-403.

19. Wojtaszewski JF, Higaki Y, Hirshman MF, Michael MD, Dufresne SD, Kahn CR, et al. Exercise modulates postreceptor insulin signaling and glucose transport in muscle-specific insulin receptor knockout mice. J Clin Invest. 1999;104(9):1257-64.

20. Aarsland A, Chinkes D, Wolfe RR. Hepatic and whole-body fat synthesis in humans during carbohydrate overfeeding. Am J Clin Nutr. 1997;65(6):1774-82.

21. Solinas G, Boren J, Dulloo AG. De novo lipogenesis in metabolic homeostasis: More friend than foe? Mol Metab. 2015;4(5):367-77.

22. Karpe F, Bickerton AS, Hodson L, Fielding BA, Tan GD, Frayn KN. Removal of triacylglycerols from chylomicrons and VLDL by capillary beds: the basis of lipoprotein remnant formation. Biochem Soc Trans. 2007;35(Pt 3):472-6.

23. Schweiger $M$, Schreiber $R$, Haemmerle $G$, Lass $A$, Fledelius $C$, Jacobsen $P$, et al. Adipose triglyceride lipase and hormone-sensitive lipase are the major enzymes in adipose tissue 
triacylglycerol catabolism. J Biol Chem. 2006;281(52):40236-41.

24. Kamp F, Hamilton JA. How fatty acids of different chain length enter and leave cells by free diffusion. Prostaglandins Leukot Essent Fatty Acids. 2006;75(3):149-59.

25. Hamilton JA, Johnson RA, Corkey B, Kamp F. Fatty acid transport: the diffusion mechanism in model and biological membranes. J Mol Neurosci. 2001;16(2-3):99-108; discussion 51-7.

26. McQuaid SE, Hodson L, Neville MJ, Dennis AL, Cheeseman J, Humphreys SM, et al. Downregulation of adipose tissue fatty acid trafficking in obesity: a driver for ectopic fat deposition? Diabetes. 2011;60(1):47-55.

27. Moitra J, Mason MM, Olive M, Krylov D, Gavrilova O, Marcus-Samuels B, et al. Life without white fat: a transgenic mouse. Genes Dev. 1998;12(20):3168-81.

28. Roden M. How free fatty acids inhibit glucose utilization in human skeletal muscle. News Physiol Sci. 2004;19:92-6.

29. Randle PJ. Regulatory interactions between lipids and carbohydrates: the glucose fatty acid cycle after 35 years. Diabetes Metab Rev. 1998;14(4):263-83.

30. Navale AM, Paranjape AN. Glucose transporters: physiological and pathological roles. Biophys Rev. 2016;8(1):5-9.

31. Felber JP, Magnenat G, Casthelaz M, Geser CA, Muller-Hess R, de Kalbermatten N, et al. Carbohydrate and lipid oxidation in normal and diabetic subjects. Diabetes. 1977;26(7):693-9.

32. Rosen ED, Spiegelman BM. Adipocytes as regulators of energy balance and glucose homeostasis. Nature. 2006;444(7121):847-53.

33. Fisher SJ, Kahn CR. Insulin signaling is required for insulin's direct and indirect action on hepatic glucose production. J Clin Invest. 2003;111(4):463-8.

34. Porcellati F, Lucidi P, Bolli GB, Fanelli CG. Thirty years of research on the dawn phenomenon: lessons to optimize blood glucose control in diabetes. Diabetes Care. 2013;36(12):3860-2.

35. Lee SJ, Shin,S.W. The Energy Metabolism behind Danwn Phenomenon and Exercise Associated Hypoglycemia in Diabetics. WCPD10; July 15-18, 2018; Edinburgh, United Kingdom2018. 36. Morimoto $\mathrm{C}$, Tsujita T, Okuda $\mathrm{H}$. Antilipolytic actions of insulin on basal and hormoneinduced lipolysis in rat adipocytes. J Lipid Res. 1998;39(5):957-62.

37. Flannery C, Dufour S, Rabol R, Shulman GI, Petersen KF. Skeletal muscle insulin resistance promotes increased hepatic de novo lipogenesis, hyperlipidemia, and hepatic steatosis in the elderly. Diabetes. 2012;61(11):2711-7.

38. Kim JK, Michael MD, Previs SF, Peroni OD, Mauvais-Jarvis F, Neschen S, et al. Redistribution of substrates to adipose tissue promotes obesity in mice with selective insulin resistance in muscle. J Clin Invest. 2000;105(12):1791-7.

39. Lee EY, Lee YH, Yi SW, Shin SA, Yi JJ. BMI and All-Cause Mortality in Normoglycemia, Impaired Fasting Glucose, Newly Diagnosed Diabetes, and Prevalent Diabetes: A Cohort Study. Diabetes Care. 2017;40(8):1026-33.

40. Salgin B, Ong KK, Thankamony A, Emmett P, Wareham NJ, Dunger DB. Higher fasting plasma free fatty acid levels are associated with lower insulin secretion in children and adults and a 
higher incidence of type 2 diabetes. J Clin Endocrinol Metab. 2012;97(9):3302-9.

41. Allard P, Delvin EE, Paradis G, Hanley JA, O'Loughlin J, Lavallee C, et al. Distribution of fasting plasma insulin, free fatty acids, and glucose concentrations and of homeostasis model assessment of insulin resistance in a representative sample of Quebec children and adolescents. Clin Chem. 2003;49(4):644-9.

42. Hulman A, Simmons RK, Brunner EJ, Witte DR, Faerch K, Vistisen D, et al. Trajectories of glycaemia, insulin sensitivity and insulin secretion in South Asian and white individuals before diagnosis of type 2 diabetes: a longitudinal analysis from the Whitehall II cohort study. Diabetologia. 2017;60(7):1252-60.

43. Tabak AG, Jokela M, Akbaraly TN, Brunner EJ, Kivimaki M, Witte DR. Trajectories of glycaemia, insulin sensitivity, and insulin secretion before diagnosis of type 2 diabetes: an analysis from the Whitehall II study. Lancet. 2009;373(9682):2215-21.

44. Yeni-Komshian $H$, Carantoni M, Abbasi F, Reaven GM. Relationship between several surrogate estimates of insulin resistance and quantification of insulin-mediated glucose disposal in 490 healthy nondiabetic volunteers. Diabetes Care. 2000;23(2):171-5.

45. Soonthornpun S, Rattarasarn C, Leelawattana R, Setasuban W. Postprandial plasma glucose: a good index of glycemic control in type 2 diabetic patients having near-normal fasting glucose levels. Diabetes Res Clin Pract. 1999;46(1):23-7.

46. Lee SJ, Shin SW. Mechanisms, Pathophysiology, and Management of Obesity. N Engl J Med. 2017;376(15):1491-2.

47. Avignon A, Radauceanu A, Monnier L. Nonfasting plasma glucose is a better marker of diabetic control than fasting plasma glucose in type 2 diabetes. Diabetes Care. 1997;20(12):1822-6. 48. Ketema EB, Kibret KT. Correlation of fasting and postprandial plasma glucose with $\mathrm{HbA} 1 \mathrm{c}$ in assessing glycemic control; systematic review and meta-analysis. Arch Public Health. 2015;73:43. 49. Jelenik T, Kaul K, Sequaris G, Flogel U, Phielix E, Kotzka J, et al. Mechanisms of Insulin Resistance in Primary and Secondary Nonalcoholic Fatty Liver. Diabetes. 2017;66(8):2241-53.

50. Sanders FW, Griffin JL. De novo lipogenesis in the liver in health and disease: more than just a shunting yard for glucose. Biol Rev Camb Philos Soc. 2016;91(2):452-68.

51. Boden G, Lebed B, Schatz M, Homko C, Lemieux S. Effects of acute changes of plasma free fatty acids on intramyocellular fat content and insulin resistance in healthy subjects. Diabetes. 2001;50(7):1612-7.

52. Stannard SR, Thompson MW, Fairbairn K, Huard B, Sachinwalla T, Thompson CH. Fasting for $72 \mathrm{~h}$ increases intramyocellular lipid content in nondiabetic, physically fit men. Am J Physiol Endocrinol Metab. 2002;283(6):E1185-91.

53. Goodpaster BH, He J, Watkins S, Kelley DE. Skeletal muscle lipid content and insulin resistance: evidence for a paradox in endurance-trained athletes. J Clin Endocrinol Metab. 2001;86(12):5755-61.

54. Johnson NA, Stannard SR, Rowlands DS, Chapman PG, Thompson CH, O'Connor $\mathrm{H}$, et al. Effect of short-term starvation versus high-fat diet on intramyocellular triglyceride accumulation and 
insulin resistance in physically fit men. Exp Physiol. 2006;91(4):693-703.

55. Kanamori K, Ihana-Sugiyama N, Yamamoto-Honda R, Nakamura T, Sobe C, Kamiya S, et al. Postprandial Glucose Surges after Extremely Low Carbohydrate Diet in Healthy Adults. Tohoku J Exp Med. 2017;243(1):35-9.

56. Westman EC, Yancy WS, Jr., Humphreys M. Dietary treatment of diabetes mellitus in the pre-insulin era (1914-1922). Perspect Biol Med. 2006;49(1):77-83.

57. Vuorinen-Markkola $\mathrm{H}$, Koivisto VA, Yki-Jarvinen $\mathrm{H}$. Mechanisms of hyperglycemia-induced insulin resistance in whole body and skeletal muscle of type I diabetic patients. Diabetes. 1992;41(5):571-80.

58. DeFronzo RA, Hendler R, Simonson D. Insulin resistance is a prominent feature of insulindependent diabetes. Diabetes. 1982;31(9):795-801.

59. Gavrilova O, Marcus-Samuels B, Graham D, Kim JK, Shulman Gl, Castle AL, et al. Surgical implantation of adipose tissue reverses diabetes in lipoatrophic mice. J Clin Invest. 2000;105(3):2718.

60. Thornalley PJ, Battah S, Ahmed N, Karachalias N, Agalou S, Babaei-Jadidi R, et al. Quantitative screening of advanced glycation endproducts in cellular and extracellular proteins by tandem mass spectrometry. Biochem J. 2003;375(Pt 3):581-92.

61. Brownlee M. Biochemistry and molecular cell biology of diabetic complications. Nature. 2001;414(6865):813-20.

62. Shin SW, Lee SJ. Ectopic fat in insulin resistance, dyslipidemia, and cardiometabolic disease. N Engl J Med. 2014;371(23):2236.

63. Beisswenger P, Ruggiero-Lopez D. Metformin inhibition of glycation processes. Diabetes Metab. 2003;29(4 Pt 2):6S95-103.

64. Zhou Z, Tang Y, Jin X, Chen C, Lu Y, Liu L, et al. Metformin Inhibits Advanced Glycation End Products-Induced Inflammatory Response in Murine Macrophages Partly through AMPK Activation and RAGE/NFkappaB Pathway Suppression. J Diabetes Res. 2016;2016:4847812.

65. Ahmad S, Shahab U, Baig MH, Khan MS, Khan MS, Srivastava AK, et al. Inhibitory effect of metformin and pyridoxamine in the formation of early, intermediate and advanced glycation endproducts. PLoS One. 2013;8(9):e72128.

66. Ahmed N, Babaei-Jadidi R, Howell SK, Beisswenger PJ, Thornalley PJ. Degradation products of proteins damaged by glycation, oxidation and nitration in clinical type 1 diabetes. Diabetologia. 2005;48(8):1590-603.

67. Thornalley PJ. Measurement of protein glycation, glycated peptides, and glycation free adducts. Perit Dial Int. 2005;25(6):522-33.

68. Jonas JC, Sharma A, Hasenkamp W, Ilkova H, Patane G, Laybutt R, et al. Chronic hyperglycemia triggers loss of pancreatic beta cell differentiation in an animal model of diabetes. J Biol Chem. 1999;274(20):14112-21.

69. Schernthaner G, Guerci B, Gallwitz B, Rose L, Nicolay C, Kraus P, et al. Impact of postprandial and fasting glucose concentrations on $\mathrm{HbA} 1 \mathrm{c}$ in patients with type 2 diabetes. Diabetes Metab. 
2010;36(5):389-94.

70. Bechlioulis A, Vakalis K, Naka KK, Bourantas CV, Papamichael ND, Kotsia A, et al. Paradoxical protective effect of central obesity in patients with suspected stable coronary artery disease. Obesity (Silver Spring). 2013;21(3):E314-21.

71. Nickols-Richardson SM, Coleman MD, Volpe JJ, Hosig KW. Perceived hunger is lower and weight loss is greater in overweight premenopausal women consuming a low-carbohydrate/highprotein vs high-carbohydrate/low-fat diet. J Am Diet Assoc. 2005;105(9):1433-7.

72. Johansson E, Lubberink M, Heurling K, Eriksson JW, Skrtic S, Ahlstrom H, et al. Whole-Body Imaging of Tissue-specific Insulin Sensitivity and Body Composition by Using an Integrated PET/MR System: A Feasibility Study. Radiology. 2018;286(1):271-8.

73. Logue J, Walker JJ, Leese G, Lindsay R, McKnight J, Morris A, et al. Association between BMI measured within a year after diagnosis of type 2 diabetes and mortality. Diabetes Care. 2013;36(4):887-93.

74. Aune D, Sen A, Prasad M, Norat T, Janszky I, Tonstad S, et al. BMI and all cause mortality: systematic review and non-linear dose-response meta-analysis of 230 cohort studies with 3.74 million deaths among 30.3 million participants. BMJ. 2016;353:i2156.

75. Bhaskaran K, Dos-Santos-Silva I, Leon DA, Douglas IJ, Smeeth L. Association of BMI with overall and cause-specific mortality: a population-based cohort study of 3.6 million adults in the UK. Lancet Diabetes Endocrinol. 2018;6(12):944-53.

76. Kwon Y, Kim HJ, Park S, Park YG, Cho KH. Body Mass Index-Related Mortality in Patients with Type 2 Diabetes and Heterogeneity in Obesity Paradox Studies: A Dose-Response Meta-Analysis. PLoS One. 2017;12(1):e0168247.

77. Bluher M, Michael MD, Peroni OD, Ueki K, Carter N, Kahn BB, et al. Adipose tissue selective insulin receptor knockout protects against obesity and obesity-related glucose intolerance. Dev Cell. 2002;3(1):25-38.

78. Bluher $M$, Kahn BB, Kahn CR. Extended longevity in mice lacking the insulin receptor in adipose tissue. Science. 2003;299(5606):572-4. 